\title{
The vertical mode method in the problems of flexural-gravity waves diffracted by a vertical cylinder
}

\author{
A.A. Korobkin ${ }^{1}$, S. Malenica ${ }^{2}$, T. Khabakhpasheva ${ }^{3}$ \\ ${ }^{1}$ School of Mathematics, University of East Anglia, Norwich, UK, \\ e-mail: a.korobkin@uea.ac.uk \\ ${ }^{2}$ Bureau Veritas, Marine \& Offshore Division - Research Department, France, \\ sime.malenica@bureauveritas.com \\ ${ }^{3}$ Lavrentyev Institute of Hydrodynamics, Novosobirsk, Russia, \\ e-mail: tana@hydro.nsc.tu
}

\begin{abstract}
The linear three-dimensional problem of ice loads acting on a vertical circular cylinder frozen in an ice cover of infinite extent is studied. The loads are caused by an uni-directional hydroelastic wave propagating in the ice cover towards the cylinder mounted to the see bottom in water of constant depth. There are no open water surfaces in this problem. The deflection of the ice cover is described by the Bernoulli-Euler equation of a thin elastic plate of constant thickness. At the contact line between the ice cover and the surface of the cylinder, some edge conditions are imposed. In this study, the edge of the ice plate is either clamped to the cylinder or has no contact with the cylinder surface, with the plate edge being free of stresses and shear forces. The water is of finite constant depth, inviscid and incompressible. The problem is solved by both the vertical mode method and using the Weber integral transform in the radial coordinate. Each vertical mode corresponds to a root of the dispersion relation for flexural-gravity waves. It is proved that these two solutions are identical for the clamped edge conditions. This result is non-trivial because the vertical modes are non-orthogonal in a standard sense, they are linearly dependent, the roots of the dispersion relation can be double and even triple, and the set of the modes could be incomplete. A general solution of the wave-cylinder interaction problem is derived by the method of vertical modes and applied to different edge conditions on the contact line. There are three conditions of solvability in this problem. It is shown that these conditions are satisfied for any parameters of the problem.
\end{abstract}

Keywords: Ice cover, vertical circular cylinder, flexural-gravity waves, edge conditions

\section{Introduction}

The linear three-dimensional problem of uni-directional hydroelastic wave propagating in an infinite ice cover towards a circular vertical cylinder in water of finite depth is solved in this paper by the Weber integral transform, as in [1], and by the vertical mode method, see [2]. The present analysis is inspired by the approach suggested in [2], where two-dimensional problems of flexural-gravity waves interacting with a vertical wall were studied by the vertical mode method. The approach of [2] was generalised in [3] to the three-dimensional problems. In this generalised approach, the vertical coordinate is separated in the solution reducing the original three-dimensional problem to a set of two-dimensional radiation and diffraction problems. Each vertical mode corresponds to a root of the dispersion relation for hydroelastic waves. Such a separation is only possible for water of constant depth, flat horizontal bottom, constant thickness of the ice sheet, and vertical solid 


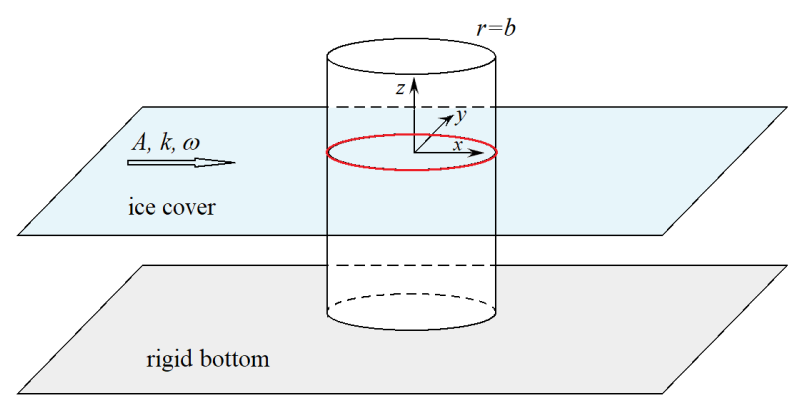

Fig. 1 Sketch of the problem and main notations.

boundaries of the flow region. The resulting two-dimensional problems are similar to radiation problems of acoustics. They are formulated for the two-dimensional Helmholtz equations with the wavenumbers equal to the roots of the dispersion relation of the hydroelastic waves, see [3]. The equations should be solved outside the cross section of the vertical cylinder with corresponding far-field conditions. The boundary conditions for the potentials are imposed on the boundary of the cylinder, where the normal derivatives of the potentials are given by linear combinations of two functions, which are independent of the roots of the dispersion relation. The vertical mode method provides a general solution of the formulated problem with two undetermined functions on the contact line between the ice cover and the vertical walls. This result is valid for any shape of the cylinder and of any number of the cylinders, see [3] for the general approach and physical meanings of the undetermined functions. One of the functions is the slope of the elastic plate deflection along the contact line, and another function provides the distribution of the shear force on the contact line. These functions are determined by using the edge conditions. The problem becomes simpler in the two-dimensional case studied in [2], where these two functions turn to two unknown numbers, and for a circular cylinder, where these functions can be represented by their Fourier series with the original problem being reduced to a set of problems for each coefficient in the series to be determined independently. Another difficulty with the vertical modes is related to their properties. It was noted in [4] that the vertical modes are non-orthogonal in a standard sense and could be incomplete. It was also shown that the modes are linearly dependent. It was shown in [3] that some roots of the dispersion relation for some parameters of the problem can be double and even triple. Therefore, the solution of this problem by the vertical mode method without its validation is questionable, strictly speaking. We are unaware of rigorous results on completeness of the vertical modes.

Similar approach to the hydroelastic wave scattering problem was employed by Evans and Porter [4]. They solved the two-dimensional scattering problem by a Green' function approach (see [4], section 3) and then by the eigen function expansion method (see [4], section 4), which is equivalent to the present method of vertical modes. They reported that the vertical mode method is much simpler to use, and it also gives useful details about the ice deflection and the flow beneath the ice. However, to validate this mode solution, they solve the same problem by another method and demonstrated that these two solutions are identical. We follow the same idea in the present paper for the problem of a vertical cylinder and the ice sheet clamped to the surface of the cylinder, see Sections 3 and 4 .

The vertical modes of ice sheet of constant thickness floating on water of finite depth were introduced in $[5,6]$ and were successfully applied to two-dimensional problems of hydroelasticity without vertical boundaries in several papers, see for example $[7,8]$. 
The effect of vertical boundaries of a flow region on deflections of infinite ice cover were studied in [9-13]. A more complicated problem of a finite ice floe frozen to a vertical cylinder in an incident wave was investigated in [14] by the vertical mode method. In [14], the modes corresponding to the two complex roots of the hydroelastic wave dispersion relation were assumed to be linearly dependent on other modes, see equation (14) in [14]. However, this fact has not been used in the analysis of [14]. A similar difficulty was encountered in [15], see section 3.2 on vertical modes and equation (3.39) which is similar to equation (14) of [14]. It is still not clear that the vertical modes taken for each root of the dispersion relation are independent of other modes and in which sense. Indeed, two of the vertical modes can be expressed as series of all other modes, see equations (49) and (50) below, but the convergence of the series do not allow us to calculate all required derivatives differentiating the series term by term. Properties of the vertical modes such as their orthogonality and linear independence have not been clarified yet. The corresponding non-selfadjoint boundary eigenvalue problem with the eigenvalue in the boundary condition still needs to be carefully analysed using the methods of functional analysis. This problem is not considered here.

\section{Formulation of the problem}

The linear problem of an incident hydroelastic wave interacting with a circular bottom-mounted cylinder is formulated with respect to a velocity potential, $\phi(x, y, z, t)$, which satisfies the Laplace equation,

$$
\nabla^{2} \phi+\phi_{z z}=0, \quad \nabla^{2} \phi=\phi_{x x}+\phi_{y y},
$$

in the flow region, $-H<z<0, r>b$, where $x=r \cos \theta, y=r \sin \theta$, and $r, \theta, z$ are the cylindrical coordinates, $H$ is the water depth and $b$ is the radius of the rigid circular cylinder, see Figure 1. The plane $z=-H$ corresponds to the flat rigid bottom and the plane $z=0$ corresponds to the ice-fluid interface. The potential $\phi$ satisfies also the boundary conditions on the bottom,

$$
\phi_{z}=0 \quad(z=-H, \quad r>b),
$$

on the surface of the circular cylinder,

$$
\phi_{r}=0 \quad(r=b, \quad-H<z<0),
$$

and on the ice/water interface,

$$
\phi_{z}=w_{t}(r, \theta, t) \quad(z=0, \quad r>b),
$$

where the equation $z=w(r, \theta, t)$ describes the deflection of the ice cover. The deflection $w(r, \theta, t)$ is governed by the Bernoulli-Euler equation of a thin elastic plate, see [15],

$$
m w_{t t}+D \nabla^{4} w=p(r, \theta, 0, t) \quad(r>b),
$$

where $m$ is the mass of the ice cover per unit area, $m=\rho_{i} h_{i}, h_{i}$ is the ice thickness, $\rho_{i}$ is the ice density, $D$ is the rigidity coefficient, $D=E h_{i}^{3} /\left[12\left(1-\nu^{2}\right)\right]$ for an elastic plate of constant thickness, $E$ is the Young module of the ice, $\nu$ is the Poisson ratio, $p$ is the hydrodynamic pressure given on the ice/water interface by the linearized Bernoulli equation,

$$
p(r, \theta, 0, t)=-\rho \phi_{t}(r, \theta, 0, t)-\rho g w(r, \theta, t),
$$

where $\rho$ is the water density and $g$ is the gravitational acceleration. 
The conditions at the contact line, $z=0$ and $r=b$, between the ice cover and the surface of the cylinder can be complicated in practical problems. These conditions will be used at the very end of the solution procedure. The clamped edge conditions,

$$
w=0, \quad w_{r}=0 \quad(r=b),
$$

will be used for a reference. These conditions imply that the ice sheet is frozen to the surface of the cylinder, see [9-14]. Free-edge conditions at the contact line will be considered in Section 5 .

The flow and ice deflection are caused by an incident hydroelastic wave,

$$
w_{\text {inc }}(x, t)=A \cos (k x-\omega t),
$$

propagating in the positive $x$-direction, see Figure 1 , where $A$ is the amplitude of the incident wave, $k$ is the wavenumber and $\omega$ is the wave frequency. The real and positive $\omega$ and $k$ in (8) are related by the dispersion equation, see [16],

$$
\omega^{2}\left(m+\frac{\rho}{k \tanh (k H)}\right)=\rho g+D k^{4},
$$

which is convenient to use in its non-dimensional form,

$$
\left(\varkappa^{4}+\delta\right) \varkappa \tanh (\varkappa)-q=0 .
$$

Here $\varkappa=k H$ is the non-dimensional wavenumber, $q=\left(\omega^{2} H / g\right)\left(H / L_{c}\right)^{4}, \delta=\left(1-\omega^{2} / \omega_{0}^{2}\right)\left(H / L_{c}\right)^{4}$, $L_{c}=(D / \rho g)^{1 / 4}$ is the characteristic length of the ice sheet and $\omega_{0}=(\rho g / m)^{1 / 2}$ is the frequency of the broken ice, see [17]. The real positive root of (9) is denoted by $k$ without index. Other roots, which are complex, are distinguished by their indexes. The velocity potential $\phi_{\text {inc }}(x, z, t)$ corresponding to the hydroelastic incident wave (8) reads

$$
\phi_{\text {inc }}(x, z, t)=A \frac{\omega}{k} \frac{\cosh [k(z+H)]}{\sinh [k H]} \sin (k x-\omega t) .
$$

The formulated problem (1) - (11) can be solved in the same way as the corresponding problem without ice cover, see [18], if the edge conditions are disregarded. The solution of the hydroelastic wave diffraction problem without these conditions can be written using only the vertical mode presented in the incident wave potential (11) without any evanescent modes corresponding to the complex roots of equation (10). The resulting velocity potential reads

$$
\phi_{0}(x, y, z, t)=A \frac{\omega}{k} \frac{\cosh [k(z+H)]}{\sinh [k H]} \Re\left[-i \Phi(r, \theta) e^{-i \omega t}\right],
$$

where $\Re$ stands for the real part of a complex number. The kinematic condition (4) and equation (12) provide the deflection of the cover as

$$
w_{0}(x, y, t)=A \Re\left[\Phi(r, \theta) e^{-i \omega t}\right] .
$$

The deflection (13) approaches the incident wave (8) in the far field if

$$
\Phi(r, \theta) \sim e^{i k r \cos \theta} \quad \text { as } \quad r \rightarrow \infty .
$$

The functions (12) and (13) satisfy the bottom condition (2), the kinematic condition (4) and the equations (5), (6) for any $\Phi(r, \theta)$. The potential $\Phi(r, \theta)$ is the solution of the boundary problem

$$
\nabla^{2} \Phi+k^{2} \Phi=0 \quad(r>b)
$$




$$
\Phi_{r}=0 \quad(r=b),
$$

which also satisfies the far-field condition (14). The boundary problem (14)-(16) is similar to the problem of linear water waves diffracted by a vertical cylinder with circular cross section [18]. The only difference of the present problem from that of linear wave diffraction is that $\omega$ and $k$ for water waves without ice are related by a dispersion relation which differs from (9) and can be obtained from (10) by letting $L_{c} / H \rightarrow 0$ and $\omega / \omega_{0} \rightarrow 0$.

By using the formula, see [19],

$$
e^{i k r \cos \theta}=\sum_{m=0}^{\infty} \epsilon_{m} i^{m} J_{m}(k r) \cos (m \theta),
$$

where $\epsilon_{m}=2$ for $m \geq 1$ and $\epsilon_{0}=1, J_{m}(k r)$ is the Bessel function of the first kind with order $m$, and the fact that the products $J_{m}(k r) \cos (m \theta)$ and $H_{m}^{(1)}(k r) \cos (m \theta)$, where $H_{m}^{(1)}(k r)$ is the Hankel function of the first kind corresponding to outward-propagating cylindrical waves, are solutions of (15), the solution of (14)-(16) can be presented in the form, see [18],

$$
\Phi(r, \theta)=\sum_{m=0}^{\infty} \epsilon_{m} i^{m}\left[J_{m}(k r)-\frac{J_{m}^{\prime}(k b)}{H_{m}^{(1)}(k b)} H_{m}^{(1)}(k r)\right] \cos (m \theta) .
$$

For a non-circular vertical cylinder [20], equation (15) should be solved in the exterior of the cylinder cross section, condition (16) should be changed to the condition of zero normal derivative, $\partial \Phi / \partial n=0$, on the cylinder, and the far-field condition (14) is used as-is.

The functions (12) and (13), where $\Phi(r, \theta)$ is given by (17), satisfy all equations of the formulated problem (1)-(9) except the edge conditions. Note that the edge conditions were not used in (17). Therefore the complete solution of the linear problem of hydroelasticity (1)-(11) is given by (12), (13) with corrections accounting for the edge conditions,

$$
\phi(x, y, z, t)=\phi_{0}(x, y, z, t)+\phi_{c}(r, \theta, z, t), \quad w(x, y, t)=w_{0}(x, y, t)+w_{c}(r, \theta, t) .
$$

The correction potential, $\phi_{c}(r, \theta, z, t)$, and the correction deflection, $w_{c}(r, \theta, t)$, are the solutions of the radiation problem with the periodic in time flow caused by given disturbances at the plate edge, $r=b$. For a cylinder frozen in the ice cover, the correction functions satisfy equations (1)-(6), describe outgoing waves at infinity, and satisfy the edge conditions,

$$
\partial w_{c} / \partial r=0, \quad w_{c}=-A \Re\left[\Phi(b, \theta) e^{-i \omega t}\right] \quad(r=b),
$$

where $\Phi(r, \theta)$ is the solution of the problem (14)-(16). Note that $\partial w_{0} / \partial r=0$ on $r=b$. On the cylinder surface, $r=b$, we have $J_{m}(k b) H_{m}^{(1) \prime}(k b)-J_{m}{ }^{\prime}(k b) H_{m}^{(1)}(k b)=2 i /(\pi k b)$, see [19], and then

$$
\Phi(b, \theta)=\frac{2 i}{\pi k b} \sum_{m=0}^{\infty} \epsilon_{m} i^{m} \frac{\cos (m \theta)}{H_{m}^{(1) \prime(k b)}} .
$$

Then the second condition in (19) can be written as

$$
w_{c}(b, \theta, t)=-\sum_{m=0}^{\infty} \Re\left[L_{m}(t)\right] \cos (m \theta), \quad L_{m}(t)=\frac{2 A}{\pi k b} \frac{\epsilon_{m} i^{m+1}}{H_{m}^{(1) \prime}(k b)} e^{-i \omega t} .
$$

predicting the following form of the correction deflection, $r>b$,

$$
w_{c}(r, \theta, t)=-\sum_{m=0}^{\infty} \Re\left[L_{m}(t) W_{m}(r)\right] \cos (m \theta),
$$


where

$$
W_{m}(b)=1, \quad W_{m}^{\prime}(b)=0 .
$$

Correspondingly the correction potential $\phi_{c}(r, \theta, z, t)$ is sought in the form

$$
\phi_{c}(r, \theta, z, t)=\sum_{m=0}^{\infty} \Re\left[i \omega L_{m}(t) \Phi_{m}(r, z)\right] \cos (m \theta),
$$

where

$$
\frac{\partial \Phi_{m}}{\partial z}(r, 0)=W_{m}(r) \quad(r>b) .
$$

The bottom condition (2) and the condition (3) on the surface of the cylinder give

$$
\frac{\partial \Phi_{m}}{\partial z}(r,-H)=0, \quad \frac{\partial \Phi_{m}}{\partial r}(b, z)=0 .
$$

It is convenient to introduce the operator in $r$, see [1], defined by

$$
S_{m}<\Phi_{m}>=\frac{\partial^{2} \Phi_{m}}{\partial r^{2}}+\frac{1}{r} \frac{\partial \Phi_{m}}{\partial r}-\frac{m^{2}}{r^{2}} \Phi_{m}
$$

Then $\nabla^{2}\left(\Phi_{m}(r, z) \cos (m \theta)\right)=S_{m}<\Phi_{m}>\cos (m \theta)$ and the Laplace equation (1) provides

$$
S_{m}<\Phi_{m}>+\Phi_{m, z z}=0 \quad(-H<z<0, r>b) .
$$

Correspondingly,

$$
\nabla^{4}\left(W_{m}(r) \cos (m \theta)\right)=S_{m}^{2}<W_{m}>\cos (m \theta)
$$

and the equations (5) and (6) give after dividing both sides of (5) by $\rho g$,

$$
L_{c}^{4} S_{m}^{2}<W_{m}>+\left(1-\frac{\omega^{2}}{\omega_{0}^{2}}\right) W_{m}=\frac{\omega^{2}}{g} \Phi_{m}(r, 0) \quad(r>b),
$$

where equations $D=\rho g L_{c}^{4}$ and $\rho g / m=\omega_{0}^{2}$ were used. At infinity, $r \rightarrow \infty$, the correction functions represent waves radiated from the cylinder. The boundary problem (21)-(27) is solved in the next section by the method of separating variables which leads to the so-called vertical modes of an elastic floating plate.

\section{Vertical-mode solution}

The plate equation (27) can be transformed to a boundary condition for equation (26), which is for the potential $\Phi_{m}(r, z)$. By using condition (23) and equation (26), we obtain

$$
S_{m}^{2}<\Phi_{m, z}>=\frac{\partial^{5} \Phi_{m}}{\partial z^{5}}(r, z)
$$

Setting $z=0$ in this equation and using condition (23), non-dimensional variables $\hat{r}=r / H, \hat{z}=$ $z / H$ and the notations from the dispersion relation (10), we can exclude $W_{m}(r)$ from $(27)$ and present (27) in the form of the following boundary condition for equation (26),

$$
\frac{\partial^{5} \Phi_{m}}{\partial \hat{z}^{5}}+\delta \frac{\partial \Phi_{m}}{\partial \hat{z}}=q \Phi_{m} \quad(\hat{z}=0, \hat{r}>\hat{b})
$$


Note that the equation (26) and the boundary conditions (24) do not change their forms in the nondimensional variables. We shall find a solution of equation (26) subject to the boundary conditions (24) and (28), which describes out-going waves as $\hat{r} \rightarrow \infty$. Note that there are no forcing terms in this problem. We need to find all possible non-trivial solutions of this problem. Then we determine the deflections $W_{m}(r)$ using (23), and finally satisfy the conditions (21), the first of which guarantees that the solution is non-trivial.

By the method of separating variables, the product $\Phi_{m}(\hat{r}, \hat{z})=R(\hat{r}) f(\hat{z})$ satisfies equation (26) and boundary conditions on the bottom $\left(24_{1}\right)$ and the ice-water interface (28) if $f(\hat{z})$ is a nontrivial solution of the following spectral problem

$$
\begin{gathered}
f^{\prime \prime}-\varkappa^{2} f=0 \quad(-1<\hat{z}<0), \\
\frac{\mathrm{d} f}{\mathrm{~d} \hat{z}}(-1)=0, \quad \frac{\mathrm{d}^{5} f}{\mathrm{~d} \hat{z}^{5}}+\delta \frac{\mathrm{d} f}{\mathrm{~d} \hat{z}}=q f(0),
\end{gathered}
$$

where $\varkappa$ is a root of the dispersion relation $(10)$, and $R(\hat{r})$ is a solution of the equation

$$
\frac{\mathrm{d}^{2} R}{\mathrm{~d} \hat{r}^{2}}+\frac{1}{\hat{r}} \frac{\mathrm{d} R}{\mathrm{~d} \hat{r}}+\left(\varkappa^{2}-\frac{m^{2}}{\hat{r}^{2}}\right) R=0 \quad(\hat{r}>\hat{b})
$$

which describes out-going waves. The solution of (29) are non-trivial if $\varkappa$ is a root of the dispersion relation (10). The solutions of (29) and (30) are convenient to normalise by the conditions $f^{\prime}(0)=1$ and $R^{\prime}(\hat{b})=1$. Note that if $\varkappa$ is a solution of the dispersion relation (10), then $-\varkappa$ is also the solution. The equation has two real roots, $\pm \varkappa_{0}, \varkappa_{0}>0$, four complex roots, $\pm a_{0} \pm i b_{0}$, where $a_{0}>0$ and $b_{0}>0$, and infinite number of pure imaginary roots, $\varkappa_{n}= \pm i æ_{n}, æ_{n+1}>æ_{n}>0$ for $n \geq 1$. Different solutions of the problem (29) are obtained if we restrict ourselves to the roots $\varkappa_{-2}=-a_{0}+i b_{0}, \varkappa_{-1}=a_{0}+i b_{0}, \varkappa_{0}$ and $\varkappa_{n}=i æ_{n}$, where the numbering of the roots from [3] is used. The corresponding solutions of (29) normalised with $f^{\prime}(0)=1$ are

$$
f_{n}(\hat{z})=\frac{\cosh \left[\varkappa_{n}(\hat{z}+1)\right]}{\varkappa_{n} \sinh \left[\varkappa_{n}\right]}
$$

where $n \geq-2$. Note that the solutions for $n \geq 0$ are real, and $f_{-2}(\hat{z})=f_{-1}^{*}(\hat{z})$, where the star stands for complex conjugate. These solutions are called the vertical modes of a floating elastic plate. These modes are non-orthogonal in a standard sense.

It is convenient to introduce the scalar product of two functions $F(\hat{z})$ and $G(\hat{z})$ defined in the interval $-1 \leq \hat{z} \leq 0$ by, see [3],

$$
<F, G>=\int_{-1}^{0} F(\hat{z}) G(\hat{z}) \mathrm{d} \hat{z}+\frac{1}{q}\left(F^{\prime \prime \prime}(0) G^{\prime}(0)+F^{\prime}(0) G^{\prime \prime \prime}(0)\right) .
$$

The vertical modes are orthogonal with respect to this scalar product, $\left\langle f_{j}, f_{n}\right\rangle=0$, where $j \neq n$. We denote $<f_{n}, f_{n}>$ by $Q_{n}$. It is not necessary that $Q_{n}$ is real and positive. By algebra,

$$
Q_{n}=\frac{1}{2 \varkappa_{n}^{2} q^{2}}\left[\varkappa_{n}^{2}\left(\varkappa_{n}^{4}+\delta\right)^{2}+q\left(5 \varkappa_{n}^{4}+\delta-q\right)\right] .
$$

For the imaginary roots of the dispersion relation, $\varkappa_{n}=i æ_{n}$, where $æ_{n}>0$ and $n \geq 1$, we have $æ_{n}=\pi n-q(\pi n)^{-5}+O\left(n^{-6}\right)$ as $n \rightarrow \infty$. Therefore, $Q_{n}=O\left(n^{8}\right)$ as $n \rightarrow \infty$.

The corresponding solutions of the equation (30) for $\varkappa=\varkappa_{n}$ normalised with $R_{n}^{(m) \prime}(\hat{b})=1$ and describing waves propagating from the cylinder are

$$
R_{n}^{(m)}(\hat{r})=\frac{H_{m}^{(1)}\left(\varkappa_{n} \hat{r}\right)}{\varkappa_{n} H_{m}^{(1) \prime}\left(\varkappa_{n} \hat{b}\right)} .
$$


For $n \geq 1$ these functions are real and decay exponentially as $\hat{r} \rightarrow \infty$, see equation 9.6.4 from [19],

$$
R_{n}^{(m)}(\hat{r})=\frac{K_{m}\left(æ_{n} \hat{r}\right)}{æ_{n} K_{m}^{\prime}\left(æ_{n} \hat{b}\right)}
$$

where $K_{m}(x)$ are modified Bessel functions.

As a result, the series

$$
\Phi_{m}(\hat{r}, \hat{z})=\sum_{n=-2}^{\infty} C_{n m} R_{n}^{(m)}(\hat{r}) f_{n}(\hat{z})
$$

satisfies equation (26) and the boundary conditions on the bottom and the ice-water interface, and describes out-going waves at infinity for arbitrary coefficients $C_{n m}$. These coefficients should be determined in such a way that the conditions (21), (23) and the second condition in (24) are satisfied.

The kinematic condition (23) and the scaling of the vertical modes provide

$$
W_{m}(r)=\frac{1}{H} \sum_{n=-2}^{\infty} C_{n m} R_{n}^{(m)}(\hat{r}), \quad r=\hat{r} H
$$

The second boundary condition on the cylinder (24) together with the equation (36) yield

$$
\frac{\partial \Phi_{m}}{\partial r}(b, z)=\frac{1}{H} \sum_{n=-2}^{\infty} C_{n m} f_{n}(\hat{z})=0 \quad(-1<\hat{z}<0) .
$$

Equation (38) does not imply, in general, that $C_{n m}=0$ for $n \geq-2$ because the system of the functions $f_{n}(\hat{z})$ is not necessary complete and the functions are not necessary independent. A care is needed also in calculations of the derivatives at the contact line, where $r=b$ and $z=0$. In particular, the second derivative $\partial\left[\left(\partial \Phi_{m} / \partial r\right)(b, z)\right] / \partial z$ is equal to zero on the cylinder, $-H<z<0$, including the limit as $z \rightarrow 0$. On the other hand, the derivative $\partial\left[\left(\partial \Phi_{m} / \partial z\right)(r, 0)\right] / \partial r=\partial W_{m} / \partial r$, in general, is not equal to zero as $r \rightarrow b$. The edge conditions (21) are understood below as the limits:

$$
\begin{gathered}
W_{m}(b)=\lim _{r \rightarrow b} \lim _{z \rightarrow 0}\left[\frac{\partial \Phi_{m}}{\partial z}(r, z)\right]=1 \\
\frac{\partial W_{m}}{\partial r}(b)=\lim _{r \rightarrow b} \lim _{z \rightarrow 0}\left[\frac{\partial}{\partial r}\left(\frac{\partial \Phi_{m}}{\partial z}\right)(r, z)\right]=\lim _{r \rightarrow b} \lim _{z \rightarrow 0}\left[\frac{\partial}{\partial z}\left(\frac{\partial \Phi_{m}}{\partial r}\right)(r, z)\right]=0 .
\end{gathered}
$$

The order of differentiation in (40) can be changed because the derivatives are calculated at a distance from the cylinder, $r=b$, and the limit $r \rightarrow b$ is taken thereafter. In the following we understand the condition (38) also as the limit $r \rightarrow b+0$, similar to (39) and (40).

To satisfy (38), (39) and (40), we use the non-dimensional variables and calculate the limit, see $[3]$

$$
\lim _{\hat{r} \rightarrow \hat{b}}<\frac{\partial \Phi_{m}}{\partial \hat{r}}(\hat{r}, \hat{z}), f_{k}(\hat{z})>,
$$

where $k \geq-2$, by using (36) and then independently by using the definition of the scalar product (32) and the conditions (38)-(40), see [2]. The series (36) and the orthogonality of the functions $f_{j}(\hat{z})$ provide

$$
\lim _{\hat{r} \rightarrow \hat{b}}<\frac{\partial \Phi_{m}}{\partial \hat{r}}(\hat{r}, \hat{z}), f_{k}(\hat{z})>=\sum_{n=-2}^{\infty} C_{n m} \frac{\mathrm{d} R_{n}^{(m)}}{\mathrm{d} \hat{r}}(\hat{b})<f_{n}, f_{k}>=C_{k} Q_{k}
$$


where $Q_{k}$ are given by (33). On the other hand, by using (32) and conditions (38) and (40), we obtain

$$
\begin{gathered}
\lim _{\hat{r} \rightarrow \hat{b}}<\frac{\partial \Phi_{m}}{\partial \hat{r}}(\hat{r}, \hat{z}), f_{k}(\hat{z})>= \\
\lim _{\hat{r} \rightarrow \hat{b}}\left[\int_{-1}^{0} \frac{\partial \Phi_{m}}{\partial \hat{r}}(\hat{r}, \hat{z}) f_{k}(\hat{z}) \mathrm{d} \hat{z}+\frac{1}{q}\left(\frac{\partial^{3}}{\partial \hat{z}^{3}}\left(\frac{\partial \Phi_{m}}{\partial \hat{r}}\right)(\hat{r}, 0) f_{k}^{\prime}(0)+\frac{\partial}{\partial \hat{z}}\left(\frac{\partial \Phi_{m}}{\partial \hat{r}}\right)(\hat{r}, 0) f_{k}^{\prime \prime \prime}(0)\right)\right],
\end{gathered}
$$

where the limit of the integral is zero due to (38), the limit of the third term is zero due to (40) and $f_{k}^{\prime}(0)=1$ due to the normalisation of the vertical modes. By using (25), (26) one can show that

$$
\frac{\partial^{3}}{\partial \hat{z}^{3}}\left(\frac{\partial \Phi_{m}}{\partial \hat{r}}\right)(\hat{r}, 0)=H^{4} \frac{\partial^{3}}{\partial z^{3}}\left(\frac{\partial \Phi_{m}}{\partial r}\right)(r, 0)=-H^{4} \frac{\partial}{\partial r}\left(S_{m}<W_{m}>\right)
$$

where the limit of this expression as $\hat{r} \rightarrow \hat{b}$ is denoted by $q H g_{m}$ with the non-dimensional constant $g_{m}$ is to be determined. Equating the results in (41) and (42) we arrive at the formulae for the coefficients $C_{n m}$,

$$
C_{n m}=\frac{H g_{m}}{Q_{n}} .
$$

The condition (39) has not been satisfied yet. Substituting (37) and (44) in (39), we find the constant $g_{m}$,

$$
g_{m}=\left(\sum_{n=-2}^{\infty} \frac{R_{n}^{(m)}(\hat{b})}{Q_{n}}\right)^{-1}
$$

The terms of the series (45) decay as $O\left(n^{-9}\right)$ when $n \rightarrow \infty$. The non-dimensional complex-valued coefficients $g_{m}$ depend on the parameters $q, \delta$ of the dispersion relation (10) and the non-dimensional radius of the cylinder $\hat{b}$.

Equations (36), (37) and (44) provide the solution

$$
W_{m}(r)=g_{m} \sum_{n=-2}^{\infty} \frac{R_{n}^{(m)}(\hat{r})}{Q_{n}}, \quad \Phi_{m}(\hat{r}, \hat{z})=g_{m} H \sum_{n=-2}^{\infty} \frac{R_{n}^{(m)}(\hat{r}) f_{n}(\hat{z})}{Q_{n}} .
$$

It can be shown by substitution that the functions (46) satisfy equations (26) and (27). The deflections $W_{m}(r)$ satisfy the edge condition (39) due to the definition (45) of the constants $g_{m}$. The edge condition (40) is satisfied only if

$$
\sum_{n=-2}^{\infty} \frac{1}{Q_{n}}=0
$$

To prove (47), we use the technique from [4]. The left-hand side of the dispersion relation (10) is denoted by $R(\varkappa)$ and then the derivative $R^{\prime}(\varkappa)$ is calculated. Using (33) it can be shown that

$$
R^{\prime}\left(\varkappa_{n}\right)=2 q^{2} \varkappa_{n} Q_{n} /\left(\varkappa_{n}^{4}+\delta\right) .
$$

The series (47) takes now the form

$$
\sum_{n=-2}^{\infty} \frac{1}{Q_{n}}=2 q \sum_{n=-2}^{\infty} \frac{\varkappa_{n}^{2} \tanh \varkappa_{n}}{R^{\prime}\left(\varkappa_{n}\right)}
$$

and can be written as a contour integral by using the residue theorem. We introduce a function $F(z)=z^{2} \tanh z / R(z)$, where $z=x+i y$, and consider the integral of this function along a closed 
rectangular contour $C_{N}: z=x \pm 2 \pi N i,|x|<\sqrt{N}$, and $z= \pm \sqrt{N}+i y,|y|<2 \pi N$. This is straightforward to show that the contour integral tends to zero as $N \rightarrow \infty$. On the other hand, the contour integral is equal to $2 \pi i$ multiplied by the sum of the residuals of $F(z)$ corresponding to the roots of $R(z), z= \pm \varkappa_{n}, n \geq-2$, inside the contour of integration by the residue theorem. As $N \rightarrow \infty$, we find

$$
0=4 \pi i \sum_{n=-2}^{\infty} \frac{\varkappa_{n}^{2} \tanh \varkappa_{n}}{R^{\prime}\left(\varkappa_{n}\right)}
$$

which proves (47). Equality (47) can be used to check the numerical accuracy of the roots of the dispersion relation (10).

To prove that the boundary condition (38) on the surface of the cylinder is satisfied with the coefficients (44), we use the same idea as above for the equality (47). We have

$$
\frac{\partial \Phi_{m}}{\partial r}(b, z)=g_{m} \sum_{n=-2}^{\infty} \frac{f_{n}(\hat{z})}{Q_{n}}=2 g_{m} q \sum_{n=-2}^{\infty} \frac{\cosh \left[\varkappa_{n}((\hat{z}+1)]\right)}{\cosh \left[\varkappa_{n}\right]} \frac{\varkappa_{n}}{R^{\prime}\left(\varkappa_{n}\right)} \quad(-1<\hat{z}<0) .
$$

It is convenient to introduce a function $G(z)=z \cosh [z(\hat{z}+1)] /[\cosh z R(z)]$ and consider its integral along the contour $C_{N}$ introduced above. It can be shown that this integral tends to zero as $N \rightarrow \infty$ and, on the other hand, it is equal to the series on the right-hand side of (48) multiplied by $4 \pi i$. This justifies that the boundary condition on the cylinder (38) is satisfied with the derived solution.

The condition (38) provides, in particular, the following relation between the vertical modes

$$
\sum_{n=-2}^{\infty} \frac{f_{n}(\hat{z})}{Q_{n}}=0 \quad(-1<\hat{z}<0)
$$

where the series converges as $O\left(n^{-4}\right)$ when $n \rightarrow \infty$. Differentiating (49) twice and using (29), we find

$$
\sum_{n=-2}^{\infty} \frac{\varkappa_{n}^{2} f_{n}(\hat{z})}{Q_{n}}=0 \quad(-1<\hat{z}<0)
$$

where the series still converges absolutely. Therefore, equations (49) and (50) can be used to express, for example, vertical modes $f_{-2}(\hat{z})$ and $f_{-1}(\hat{z})$ through all other modes $f_{n}(\hat{z})$ starting from $n=0$. This fact is interesting, however it could be of little use because the products $R_{n}^{(m)}(\hat{r}) f_{n}(\hat{z})$, where $n \geq-2$, make the solution but not the vertical modes on their own. All the products for $n \geq-2$ are independent and should be included into the solution even if some of the vertical modes are linearly dependent of others. It would be wrong to start in the series (36) from $n=0$ on the base of (49) and (50). Note that the relations (49) and (50) were mentioned in both [14] and [15], however all vertical modes starting from $n=-2$ were included in the solutions. More details about eigenvalue problems with high-order derivatives in boundary conditions can be found in [21], chapter 4.

The obtained solution in the form of the series (46) satisfies both the equations of the problem and boundary and edge conditions. The series in (46) and the series for the required derivatives of these functions absolutely converge. The only problem left is the problem of the uniqueness of the obtained solution. To justify that the solution derived in this section is complete, the problem for the correction functions, $\phi_{c}(r, \theta, z, t)$ and $w_{c}(r, \theta, t)$ is solved in the next section by the method of integral Weber transform employed in [1]. 


\section{Solution by the Weber transform}

The boundary-value problem (20)-(27) for the correction deflections $W_{m}(r)$ and the correction potentials $\Phi_{m}(r, z)$, where $m \geq 0$, is solved in this section by using the integral Weber transform in the radial coordinate $r$, see [1]. Then the residue theorem is applied to the obtained solution, see [22] and [23], to prove that this solution is identical to that derived in Section 3 by the vertical-mode method.

At infinity, $r \rightarrow \infty$, the correction functions represent waves radiated from the cylinder. These waves, with their complex amplitudes $A_{m}$ being unknown in advance, decay as $r^{-1 / 2}$ with the distance $r$ from the cylinder. We separate the wave parts in the solution,

$$
\Phi_{m}(r, z)=A_{m} \frac{\cosh [k(z+H)]}{k \sinh (k H)} H_{m}^{(1)}(k r)+\Phi_{m}^{(e)}(r, z), \quad W_{m}(r)=A_{m} H_{m}^{(1)}(k r)+W_{m}^{(e)}(r) .
$$

The subscript $(e)$ stands for evanescent parts of the solution, which decay at infinity quicker than $r^{-1 / 2}$. The Weber transform cannot be applied to the correction functions, $\Phi_{m}(r, z)$ and $W_{m}(r)$, see the deflection of the Weber transform by (59) and (60) below, because they decay too slow at infinity but the transform can be applied to the evanescent parts of the solutions. The new unknown functions $\Phi_{m}^{(e)}(r, z)$ and $W_{m}^{(e)}(r)$ should satisfy equations (26) and (27), where $r>b$, and the bottom condition, $\partial \Phi_{m}^{(e)} / \partial z=0$, where $z=-H$, see first conditions (24). On the surface of the cylinder, the second condition in (24) and equations (51) give

$$
\frac{\partial \Phi_{m}^{(e)}}{\partial r}(b, z)=-A_{m} \frac{\cosh [k(z+H)]}{\sinh (k H)} H_{m}^{(1) \prime}(k b) .
$$

The edge conditions (21) provide

$$
W_{m}^{(e)}(b)=1-A_{m} H_{m}^{(1)}(k b), \quad W_{m}^{(e) \prime}(b)=-A_{m} k H_{m}^{(1) \prime}(k b) .
$$

At infinity,

$$
\Phi_{m}^{(e)} \sqrt{r} \rightarrow 0, \quad W_{m}^{(e)}(r) \sqrt{r} \rightarrow 0 \quad(r \rightarrow \infty) .
$$

The notations from Section 3 are used below. Also we introduce $\mu_{m}=H_{m}^{(1) \prime}\left(\varkappa_{0} \hat{b}\right)$ to make the formulae more compact. The second edge condition in (53) shows that it is convenient to introduce new unknown functions $\Phi_{m}^{(i)}(r, z)$ and $W_{m}^{(i)}(r)$ by

$$
\Phi_{m}^{(e)}(r, z)=-A_{m} \mu_{m} k \Phi_{m}^{(i)}(r, z), \quad W_{m}^{(e)}(r)=-A_{m} \mu_{m} k W_{m}^{(i)}(r) .
$$

The new unknown functions satisfy equations (26), (27), boundary conditions $\left(24_{1}\right)$ and (23), and the far-field conditions (54). The conditions $\left(24_{2}\right)$ and $\left(21_{2}\right)$ read

$$
\begin{gathered}
\frac{\partial \Phi_{m}^{(i)}}{\partial r}(b, z)=\frac{\cosh [k(z+H)]}{k \sinh [k H]} \quad(-H<z<0), \\
\frac{d W_{m}^{(i)}}{d r}(b)=1 .
\end{gathered}
$$

The condition $\left(29_{1}\right)$ gives the equation for the amplitude $A_{m}$,

$$
A_{m}\left[H_{m}^{(1)}(k b)-\mu_{m} k W_{m}^{(i)}(b)\right]=1 .
$$


The functions $\Phi_{m}^{(i)}(r, z)$ and $W_{m}^{(i)}(r)$ are obtained by using the Weber transform, see [1], [22], [23], denoted by long tilde,

$$
\widetilde{W_{m}^{(i)}}(s)=\int_{b}^{\infty} r W_{m}^{(i)}(r) Z_{m}(r, s) \mathrm{d} r,
$$

where

$$
Z_{m}(r, s)=J_{m}(s r) Y_{m}^{\prime}(s b)-J_{m}{ }^{\prime}(s b) Y_{m}(s r), \quad Z_{m}(b, s)=\frac{2}{\pi s b}, \quad \frac{\partial Z_{m}}{\partial r}(b, s)=0,
$$

$Z_{m}(r, s) \rightarrow 0$ as $r \rightarrow \infty$. The transform (59) is defined for functions in $r>b$, which decay as $\sqrt{r} W_{m}^{(i)}(r) \rightarrow 0$ where $r \rightarrow \infty$, see (54). The inverse transform reads

$$
W_{m}^{(i)}(r)=\int_{0}^{\infty} \widetilde{W_{m}^{(i)}}(s) s Z_{m}(r, s) D_{m}^{-1}(s) \mathrm{d} s,
$$

where $D_{m}(s)=\left[J_{m}^{\prime}(s b)\right]^{2}+\left[Y_{m}^{\prime}(s b)\right]^{2}$. We shall determine $\widetilde{W_{m}^{(i)}}(s)$ and show that this function is real and odd for real and positive $s$, see (61). Then we shall determine the deflection $W_{m}^{(i)}(r)$ by using the inverse transform (61) and finally find the potential $\Phi_{m}^{(i)}(r, z)$. Note that the present method is focused on the deflection but the method of vertical modes is focused on the potential of the flow beneath the ice, see Section 3 .

The Weber transform (59) is applied to equations (26) and (27) written for $\left.W_{m}^{(i)} r\right)$ and $\Phi_{m}^{(i)}(r, z)$, see (51) and (55). We use the formulas

$$
\begin{aligned}
& S_{m} \widetilde{<f(r)}>(s)=-s^{2} \tilde{f}(s)-\frac{2}{\pi s} f^{\prime}(b), \\
& S_{m}^{2} \widetilde{<f(r)}>(s)=s^{4} \tilde{f}(s)+\frac{2 s}{\pi} f^{\prime}(b)-\frac{2}{\pi s} \frac{d}{d r}\left(S_{m}<f(r)>\right)_{r=b}
\end{aligned}
$$

from [1] which are valid for any function $f(r)$ defined in $r>b$ and decaying at infinity quicker than $O\left(r^{-\frac{1}{2}}\right)$. Then equation (26), $S_{m}<\Phi_{m}^{(i)}>+\Phi_{m, z z}^{(i)}=0$, provides

$$
-s^{2} \widetilde{\Phi_{m}^{(i)}}+\Phi_{m, z z}^{(i)}=\frac{2}{\pi s} \Phi_{m, r}^{(i)}(b, z) \quad(-H<z<0),
$$

and the equation (27) gives

$$
L_{c}^{4}\left\{s^{4} \widetilde{W_{m}^{(i)}}(s)+\frac{2 s}{\pi} W_{m, r}^{(i)}(b)-\frac{2}{\pi s} \frac{d}{d r}\left(S_{m}<W_{m}^{(i)}(r)>\right)_{r=b}\right\}+\left(1-\frac{\omega^{2}}{\omega_{0}^{2}}\right) \widetilde{W_{m}^{(i)}}(s)=\frac{\omega^{2}}{g} \widetilde{\Phi_{m}^{(i)}}(s, 0) .
$$

The condition (23) on the ice/water interface and the condition $\left(24_{1}\right)$ on the bottom yield

$$
\widetilde{\Phi_{m, z}^{(i)}}(s, 0)=\widetilde{W_{m}^{(i)}}(s), \quad \widetilde{\Phi_{m, z}^{(i)}}(s,-H)=0 .
$$

The right-hand side in (63) is calculated by using (56),

$$
\frac{2}{\pi s} \Phi_{m, r}^{(i)}(b, z)=\frac{P_{m}}{s} \cosh [k(z+H)], \quad P_{m}=\frac{2}{\pi k \sinh (k H)} .
$$


The solution of the boundary problem (63), (65) and (66) reads

$$
\widetilde{\Phi_{m}^{(i)}}(s, z)=C_{1}(s) \cosh [s(z+H)]+C_{2}(s) \cosh [k(z+H)]
$$

where

$$
C_{2}=\frac{P_{m}}{s\left(k^{2}-s^{2}\right)}, \quad C_{1}(s)=\frac{\widetilde{W_{m}^{(i)}}(s)}{s \sinh (s H)}-\frac{P_{m}}{s\left(k^{2}-s^{2}\right)} \cdot \frac{k \sinh (k H)}{s \sinh (s H)} .
$$

In particular, on the ice/water interface, $z=0$,

$$
\widetilde{\Phi_{m}^{(i)}}(s, 0)=\frac{\widetilde{W_{m}^{(i)}}(s)}{s \tanh (s H)}+\frac{P_{m} \cosh k H}{s\left(k^{2}-s^{2}\right)}\left(1-\frac{k \tanh (k H)}{s \tanh (s H)}\right) .
$$

Substituting (69) in (64) and denoting $\frac{d}{d r}\left(S_{m}<W_{m}^{(i)}>\right)_{r=b}$ by $V_{m}$, we arrive at the following equation for $\widetilde{W_{m}^{(i)}}(s)$ :

$$
\frac{\widetilde{W_{m}^{(i)}}(s) R(s H)}{s H \tanh (s H)}\left(\frac{L_{c}}{H}\right)^{4}=\frac{\omega^{2} P_{m} \cosh (k H)}{g s\left(k^{2}-s^{2}\right)}\left(1-\frac{k \tanh (k H)}{s \tanh (s H)}\right)+\frac{2}{\pi s} L_{c}^{4}\left(V_{m}-s^{2}\right),
$$

where $s$ is the real positive parameter of the Weber transform. The left-hand side in (70) is equal to zero at $s=k, R(k H)=R\left(\varkappa_{0}\right)=0$, see the dispersion relation $(9)$. To avoid singularity of $\widetilde{W_{m}^{(i)}}(s)$ at $s=k$, we require that the right-hand side in (70) is also equal to zero at $s=k$. This gives the equation for $V_{m}$ :

$$
\begin{aligned}
& L_{c}^{4} \cdot \frac{2}{\pi k} V_{m}=\lim _{s \rightarrow k}\left\{L_{c}^{4} \frac{2 s}{\pi}-\frac{\omega^{2}}{g} \frac{P_{m} \cosh (k H)}{s\left(k^{2}-s^{2}\right)}\left(1-\frac{k \tanh (k H)}{s \tanh (s H)}\right)\right\} \\
& =\frac{2 k}{\pi} L_{c}^{4}-\frac{\omega^{2}}{g k \cdot 2 k} \frac{2}{\pi k \tanh (k H)} \lim _{s \rightarrow k}\left[\frac{1}{k-s}\left(1-\frac{k \tanh (k H)}{s \tanh (s H)}\right)\right],
\end{aligned}
$$

where

$$
\lim _{s \rightarrow k}\left[\frac{1}{k-s}\left(1-\frac{k \tanh (k H)}{s \tanh (s H)}\right)\right]=-\frac{1}{k}\left(1+\frac{2 k H}{\sinh (2 k H)}\right) .
$$

By using the dispersion relation (9) and the notations from (10), one finds

$$
H^{2} V_{m}=\varkappa_{0}^{2}+\frac{1+2 \varkappa_{0} / \sinh \left(2 \varkappa_{0}\right)}{\varkappa_{0}^{2}\left(\varkappa_{0}^{4}+\delta\right)}
$$

and then (70) provides

$$
\begin{gathered}
\widetilde{s W_{m}^{(i)}}(s)=\left\{-\frac{2}{\pi} L_{c}^{4}\left(s^{2}-k^{2}\right)+\frac{2}{\pi} \frac{\omega^{2}}{g k \cdot \tanh (k H)}\left[\frac{1-k \tanh (k H) /(s \tanh (s H))}{k^{2}-s^{2}}\right.\right. \\
\left.\left.+\frac{1}{2 k^{2}}\left(1+\frac{2 k H}{\sinh (2 k H)}\right)\right]\right\} \times \frac{s \tanh (s H)}{s \tanh (s H)\left[L_{c}^{4} s^{4}+\left(1-\omega^{2} / \omega_{0}^{2}\right)\right]-\omega^{2} / g}=\frac{2 H^{2}}{\pi} \frac{F(\xi)}{R(\xi)},
\end{gathered}
$$

where

$$
\begin{gathered}
F(\xi)=\left(\varkappa_{0}^{2}-\xi^{2}+\frac{\varkappa_{0}^{4}+\delta}{\varkappa_{0}^{2}-\xi^{2}}+C\left(\varkappa_{0}\right)\right) \xi \tanh \xi-\frac{q}{\varkappa_{0}^{2}-\xi^{2}}, \\
R(\xi)=\left(\xi^{4}+\delta\right) \xi \tanh \xi-q, \quad \xi=s H, \quad \varkappa_{0}=k H
\end{gathered}
$$




$$
C\left(\varkappa_{0}\right)=\frac{\varkappa_{0}^{4}+\delta}{2 \varkappa_{0}^{2}}\left(1+\frac{2 \varkappa_{0}}{\sinh \left(2 \varkappa_{0}\right)}\right), \quad F\left(\varkappa_{0}\right)=0, \quad R\left(\varkappa_{0}\right)=0
$$

Note that both $F(\xi)$ and $R(\xi)$ are even functions of $\xi$. Substituting (72) in (61) and using the following formulae

$$
\frac{Z_{m}(r, s)}{D_{m}(s)}=\frac{i}{2}\left[\frac{H_{m}^{(1)}(s r)}{H_{m}^{(1)^{\prime}}(s b)}-c . c .\right]=-\Im\left[\frac{H_{m}^{(1)}(s r)}{H_{m}^{(1) \prime}(s b)}\right], \quad \frac{Z_{m}(r,-s)}{D_{m}(-s)}=\frac{Z_{m}(r, s)}{D_{m}(s)},
$$

where $s>0$, see [22], equation (5.6), and [23], equation (51), and $\Im$ stands for the imaginary part of a complex number, we find

$$
W_{m}^{(i)}(r)=-\frac{H}{\pi} \Im\left(\int_{-\infty}^{\infty} \frac{F(\xi)}{R(\xi)} \frac{H_{m}^{(1)}(\xi \hat{r})}{H_{m}^{(1) \prime}(\xi \hat{b})} \mathrm{d} \xi\right)
$$

Here the derivative $H_{m}^{(1) \prime}(z \hat{b})$ has no zeros in the upper half-plane, $\Im z>0$, see figure 9.6 in [19]. Also

$$
\frac{H_{m}^{(1)}(z \hat{r})}{H_{m}^{(1)_{\prime}(z \hat{b})}} \sim \frac{1}{i} \sqrt{\frac{\hat{b}}{\hat{r}}} e^{i z(\hat{r}-\hat{b})} \quad(|z| \rightarrow \infty, \quad 0<\arg z<\pi) .
$$

The product $z \widetilde{W_{m}^{(i)}}(z)$ defined by $(72)$ for real and positive $z$ is continued to the upper half-plane $\Im z>0$. The continued function decays at infinity, $|z| \rightarrow \infty$, and has simple poles at $z=\varkappa_{n}$, where $n=-2,-1,1,2,3, \ldots$ and $\varkappa_{n}$ are the roots of the dispersion relation $(10), R\left(\varkappa_{n}\right)=0$. Note that $R\left(\varkappa_{0}\right)=0$ but $z=\varkappa_{0}$ is not a pole of the integrand in $(76)$ because $F\left(\varkappa_{0}\right) / R\left(\varkappa_{0}\right) \neq 0$. The integral (76) is evaluated by contour integration in the upper half-plahe $\Im z>0$ and using the residue theorem,

$$
\int_{-\infty}^{\infty} \frac{F(\xi)}{R(\xi)} \frac{H_{m}^{(1)}(\xi \hat{r})}{H_{m}^{(1) \prime}(\xi \hat{b})} \mathrm{d} \xi=2 \pi i \sum_{n=-2, n \neq 0}^{\infty} \frac{F\left(\varkappa_{n}\right)}{R^{\prime}\left(\varkappa_{n}\right)} \frac{H_{m}^{(1)}\left(\varkappa_{n} \hat{r}\right)}{H_{m}^{(1) \prime}\left(\varkappa_{n} \hat{b}\right)}
$$

Using the functions (34), we obtain

$$
W_{m}^{(i)}(r)=-2 H \Re\left(\sum_{n=-2, n \neq 0}^{\infty} \frac{\varkappa_{n} F\left(\varkappa_{n}\right)}{R^{\prime}\left(\varkappa_{n}\right)} R_{n}^{(m)}(\hat{r})\right) .
$$

The terms of the series in (77) are real for $n \geq 1$, see (35), (73) and (74). For $n=-2$ and $n=-1$ we use the relations $\varkappa_{-2}=-\overline{\varkappa_{-1}}$,

$$
F(-z)=F(z), \quad R^{\prime}(-z)=-R^{\prime}(z), \quad F(\bar{z})=\overline{F(z)}, \quad R^{\prime}(\bar{z})=\overline{R^{\prime}(z)}, \quad R_{-2}^{(m)}(\hat{r})=\overline{R_{-1}^{(m)}(\hat{r})}
$$

which provide that the series in (77) is real and, therefore, the operation $\Re$ can be dropped. Substituting (77) and (55) in (51), we find

$$
W_{m}(r)=A_{m} \mu_{m} \varkappa_{0}\left(R_{0}^{(m)}(\hat{r})+\sum_{n=-2, n \neq 0}^{\infty} \frac{2 \varkappa_{n} F\left(\varkappa_{n}\right)}{R^{\prime}\left(\varkappa_{n}\right)} R_{n}^{(m)}(\hat{r})\right),
$$

where the amplitude $A_{m}$ of the outgoing wave is defined by the condition $W_{m}(b)=1$, see $(58)$. 
Equations (73) and (74), where $\xi=\varkappa_{n}, n \neq 0$, yield

$$
R\left(\varkappa_{n}\right)=0, \quad R^{\prime}\left(\varkappa_{n}\right)=2 q^{2} \varkappa_{n} Q_{n} /\left(\varkappa_{n}^{4}+\delta\right), \quad F\left(\varkappa_{n}\right)=q\left(2 \varkappa_{0}^{2}+C\left(\varkappa_{0}\right)\right) /\left(\varkappa_{n}^{4}+\delta\right),
$$

and then

$$
\frac{2 \varkappa_{n} F\left(\varkappa_{n}\right)}{R^{\prime}\left(\varkappa_{n}\right)}=\frac{2 \varkappa_{0}^{2}+C\left(\varkappa_{0}\right)}{q Q_{n}}
$$

where $Q_{n}, n \geq-2$ are given by (33). It can be shown by using (75) and (74) that $2 \varkappa_{0}^{2}+C\left(\varkappa_{0}\right)=q Q_{0}$ and finally

$$
\frac{2 \varkappa_{n} F\left(\varkappa_{n}\right)}{R^{\prime}\left(\varkappa_{n}\right)}=\frac{Q_{0}}{Q_{n}}
$$

Substituting (79) in (78), we find

$$
W_{m}(r)=A_{m} \mu_{m} \varkappa_{0} Q_{0} \sum_{n=-2}^{\infty} \frac{R_{n}^{(m)}(\hat{r})}{Q_{n}} .
$$

Here $A_{m} \mu_{m} \varkappa_{0} Q_{0}=g_{m}$ which follows from the condition $W_{m}(b)=1$. Therefore, the deflection (80) obtained by the Weber transform and the deflection (46) obtained by the vertical-mode method are identical.

The method of the vertical modes is simpler that the method of this section. The vertical-mode method can be used to solve problems for a circular cylinder with different conditions on the contact line between the ice plate and the surface of the cylinder.

\section{Ice plate with free-free edge at the cylinder}

The problem (1)-(9) of a linear hydroelastic incident wave interacting with a single vertical circular

cylinder, which was formulated in Section 2, is considered here for the free-free conditions on the contact line between the ice plate and the surface of the cylinder being. Only the edge conditions (7) are different now. They are changed to

$$
V_{r}(b, \theta, t)=0, \quad M_{r}(b, \theta, t)=0
$$

where

$$
\begin{gathered}
V_{r}=Q_{r}+\frac{1}{r} \frac{\partial M_{r \theta}}{\partial \theta}, \quad Q_{r}=-D \frac{\partial}{\partial r}\left(\nabla^{2} w\right), \quad M_{r \theta}=-D(1-\nu) \frac{\partial}{\partial r}\left(\frac{1}{r} \frac{\partial w}{\partial \theta}\right), \\
M_{r}=-D\left(\frac{\partial^{2} w}{\partial r^{2}}+\nu\left(\frac{1}{r} \frac{\partial w}{\partial r}+\frac{1}{r^{2}} \frac{\partial^{2} w}{\partial \theta^{2}}\right)\right),
\end{gathered}
$$

$V_{r}$ is the Kelvin-Kirchhoff edge reaction, $Q_{r}$ is the transverse shearing force, $M_{r \theta}$ is the twisting moment and $M_{r}$ is the bending moment, see [24].

The solution of the formulated problem is sought in the form (18) where $w_{0}(r, \theta, t)$ and $\phi_{0}(r, \theta, z, t)$ are given by (12), (13) and (17). The correction deflection and potential, $w_{c}(r, \theta, t)$ and $\phi_{c}(r, \theta, z, t)$, are sought in the forms (20) and (22), where $W_{m}(r)$ and $\Phi_{m}(r, z)$ satisfy $(23),(24),(26)$ and (27). The conditions at $r=b$ follow from (81). The condition $M_{r}(b, \theta, t)=0$ yields

$$
W_{m}^{\prime \prime}+\frac{\nu}{b} W_{m}^{\prime}-\frac{\nu m^{2}}{b^{2}} W_{m}=-k^{2}-\frac{m^{2}}{b^{2}}(\nu-1),
$$


and the condition $V_{r}(b, \theta, t)=0$ yields

$$
W_{m}^{\prime \prime \prime}+\frac{1}{b} W_{m}^{\prime \prime}-\frac{1+(2-\nu) m^{2}}{b^{2}} W_{m}^{\prime}+\frac{(3-\nu) m^{2}}{b^{3}} W_{m}=\frac{(1-\nu) m^{2}}{b^{3}} .
$$

The notations and formulae are the same as in the Section 2 except the edge conditions at $r=b$.

The analysis of Section 3 up to equation (39) is independent of edge conditions and can be used in the present problem with free-free edge as well. We conclude that the solutions, $W_{m}(r)$ and $\Phi_{m}(r, z)$, are given by (36) and (37), where the coefficients $C_{n}$ are given by (41) and (42). However, now both the second and third terms in (42) are not zero. We denote

$$
\frac{\partial^{3}}{\partial \hat{z}^{3}}\left(\frac{\partial \Phi_{m}}{\partial \hat{r}}\right)(\hat{b}, 0)=q H g_{m}, \quad \frac{\partial}{\partial \hat{z}}\left(\frac{\partial \Phi_{m}}{\partial \hat{r}}\right)(\hat{b}, 0)=q H \nu_{m},
$$

where $g_{m}$ and $\nu_{m}$ are to be determined as part of the solution. Then equations (41) and (42) provide

$$
C_{n m}=H\left(g_{m}+\nu_{m} \varkappa_{n}^{2}\right) / Q_{n},
$$

and the equation (37) gives the deflection as

$$
\begin{gathered}
W_{m}(r)=g_{m} W_{m}^{(1)}(r)+\nu_{m} W_{m}^{(2)}(r) \\
W_{m}^{(1)}(r)=\sum_{n=-2}^{\infty} \frac{R_{n}^{(m)}(\hat{r})}{Q_{n}}, \quad W_{m}^{(2)}(r)=\sum_{n=-2}^{\infty} \frac{\varkappa_{n}^{2} R_{n}^{(m)}(\hat{r})}{Q_{n}} .
\end{gathered}
$$

The formula (86) is valid for any edge conditions. The functions $W_{m}^{(1)}(r)$ and $W_{m}^{(2)}(r)$ are independent of the type of edge conditions. Note that $W_{m}^{(1)}(r)$ appeared in the solution of the problem with clamped edge, see equation (46). In particular, $W_{m}^{(1) \prime}(b)=0$, see (47). Substituting (86) and (87) in (85), we find that the following equalities should be hold,

$$
\sum_{n=-2}^{\infty} \frac{\varkappa_{n}^{2}}{Q_{n}}=q, \quad \sum_{n=-2}^{\infty} \frac{\varkappa_{n}^{4}}{Q_{n}}=0 .
$$

These equations should be checked numerically together with (47), in order to validate numerical calculations. All information about edge conditions contains in the coefficients $g_{m}$ and $\nu_{m}$. Equations for these coefficients are obtained by substituting (86) in the edge conditions.

Substituting (86) in (83) and (84), using (30) for the derivatives of $R_{n}^{(m)}(\hat{r})$ and equalities (47) and (88), we obtain the equations with respect to $g_{m}$ and $\nu_{m}$,

$$
\begin{gathered}
\left(\frac{(1-\nu) m^{2}}{\hat{b}^{3}} L_{0}^{(m)}-q\right) g_{m}+\left(\frac{(1-\nu) m^{2}}{\hat{b}^{3}} L_{1}^{(m)}+\frac{(\nu-1) m^{2}}{\hat{b}^{2}} q\right) \nu_{m}=\frac{1-\nu}{\hat{b}^{3}} m^{2} \\
\left(\frac{(1-\nu) m^{2}}{\hat{b}^{2}} L_{0}^{(m)}-L_{1}^{(m)}\right) g_{m}+\left(\frac{(1-\nu) m^{2}}{\hat{b}^{2}} L_{1}^{(m)}-L_{2}^{(m)}+\frac{\nu-1}{\hat{b}} q\right) \nu_{m}=-\varkappa_{0}^{2}+\frac{1-\nu}{\hat{b}^{2}} m^{2}
\end{gathered}
$$

where

$$
L_{p}^{(m)}=\sum_{n=-2}^{\infty} \frac{\varkappa_{n}^{2 p}}{Q_{n}} R_{n}^{(m)}(\hat{b}) \quad(p=0,1,2), \quad l_{p}=\sum_{n=-2}^{\infty} \frac{\varkappa_{n}^{2 p}}{Q_{n}} .
$$

The system of linear equations (89) and (90) can be simplified. For $m=0$ the system gives 


$$
g_{0}=0, \quad \nu_{0}=\frac{\varkappa_{0}^{2}}{L_{2}^{(0)}+(1-\nu) q / \hat{b}} .
$$

For $m \geq 1$ the system takes the form

$$
\begin{gathered}
\left(L_{0}^{(m)}-\frac{q \hat{b}^{3}}{(1-\nu) m^{2}}\right) g_{m}+\left(L_{1}^{(m)}-\hat{b} q\right) \nu_{m}=1, \\
\left(L_{1}^{(m)}-\hat{b} q\right) g_{m}+\left(L_{2}^{(m)}-\frac{(1-\nu)\left(m^{2}-1\right)}{\hat{b}} q\right) \nu_{m}=\varkappa_{0}^{2} .
\end{gathered}
$$

The coefficients $g_{m}$ and $\nu_{m}$ do not decay as $m \rightarrow \infty$. Indeed, the asymptotic behaviour of the Bessel functions with large order, see [19], provides

$$
R_{n}^{(m)}(\hat{b}) \sim-\frac{\hat{b}}{m} \quad(m \rightarrow \infty),
$$

and then

$$
L_{p}^{(m)} \sim-\frac{\hat{b}}{m} l_{p} \quad(m \rightarrow \infty),
$$

where, $l_{0}=0, l_{2}=0$ and $l_{1}=q$, see (47) and (88). We conclude that

$$
L_{0}^{(m)} \sim-q \hat{b}^{3} /\left(2 m^{3}\right), \quad L_{2}^{(m)}=o\left(m^{-1}\right), \quad L_{1}^{(m)} \sim \hat{b} q / m \quad(m \rightarrow \infty) .
$$

The obtained asymptotic formulae and the equations (90), (93) yield

$$
g_{m} \sim-\frac{2 m^{3}}{q \hat{b}^{3}} \frac{1-\nu}{5-\nu}, \quad \nu_{m} \sim \frac{2 m}{q \hat{b}} \frac{1}{5-\nu}
$$

as $\nu \rightarrow \infty$. A special care is needed to solve the system (93) and (94) for large $m$.

Equations (86), (87), (93) and (94) solve the problem of incident hydroelastic wave diffraction by a vertical circular cylinder which is not connected to the ice plate.

\section{Numerical results}

The hydroelastic behaviour of the ice cover around a circular cylinder in an incident linear wave is investigated numerically for a sea ice with density $\rho_{i}=917 \mathrm{~kg} / \mathrm{m}^{3}$, Young 's modulus $E=4.2 \times 10^{9}$ $\mathrm{N} / \mathrm{m}^{2}$, Poisson's ratio $\nu=0.33$ and thickness $1.5 \mathrm{~m}$. The mass of the ice sheet per unit area is $m=1375.5 \mathrm{~kg} / \mathrm{m}^{2}$. The water density is $\rho=1026 \mathrm{~kg} / \mathrm{m}^{3}$, water depth is $H=15 \mathrm{~m}$. The characteristic length of this ice plate is $L_{c}=19.05 \mathrm{~m}$, and the frequency of broken ice is $\omega_{0}=2.7$ $1 / \mathrm{s}$.

The calculations were performed for $0<k H<1.4$, where all roots of the dispersion relation (10) are single and the real part of $\varkappa_{-1}$ is not small. Cases, where some roots are either close to each other or are double/triple, are not considered here. For example, there is a triple root of the dispersion relation at $\varkappa \approx 2.28 i$ for $q \approx 79.47$ and $\delta \approx 2.89$. These values correspond to the wave frequency $\omega=2.281 / \mathrm{s}$ and ice thickness $50 \mathrm{~cm}$. The conditions of double and triple roots of the dispersion relation (10) were studied in [3]. It is not clear how to define the vertical modes, see Section 3, in the case of double/triple roots of the dispersion relation (10). 


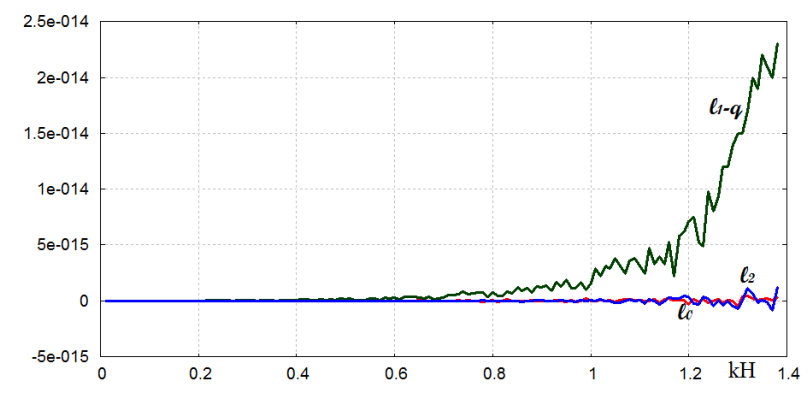

Fig. 2 The series $l_{0}(k H), l_{1}(k H)-q$ and $l_{2}(k H)$.

The roots of the dispersion relations are computed for $\varkappa_{0}=k H$ starting from 0.01 with step 0.01 . For each value of $\varkappa_{0}$, we calculate $\omega$ using the dispersion relation (9) and the parameters $q$ and $\delta$. For small $\varkappa_{0}$, which is for long waves, the frequencies $\omega$ and the dimensionless parameter $q$ are small and $\delta$ is positive. The following asymptotic formula for the complex root $\varkappa_{-1}$ can be derived

$$
\begin{gathered}
\varkappa_{-1}=\varkappa_{-10}+q \varkappa_{-11}+q^{2} \varkappa_{-12}+O\left(q^{3}\right) \quad(q \rightarrow 0), \\
\varkappa_{-10}=\delta^{\frac{1}{4}}(1+i) / \sqrt{2}, \quad \varkappa_{-11}=-\frac{1}{4 \delta \tanh \left(\varkappa_{-10}\right)}, \\
\varkappa_{-12}=-\varkappa_{-11}^{2}\left(\frac{1}{\tanh \left(\varkappa_{-10}\right) \cosh ^{2}\left(\varkappa_{-10}\right)}+\frac{5}{2 \varkappa_{-10}}\right) .
\end{gathered}
$$

This formula provides initial guess for the root $\varkappa_{-1}$ when $k H=0.01$. A better approximation of this root is obtained by the Newton method. The iterations stop when the distance between two successive approximations of the dimensionless root is less than $10^{-14}$. Then we make a step in $k H$ and take the value of the root from the previous step as the initial guess. Note that $\varkappa_{0}=k H$. In the regular case of all single roots and $\delta>0$, the pure imaginary roots $\varkappa_{n}, n \geq 1$, are close to $\pi n i$ and can be calculated by an iteration method. To validate the numerical values of the roots, we calculate the series (47) and (88). They are denoted as $l_{p}$ in (91). These series are shown in Figure 2 as functions of $k H$. It is seen that the accuracy of the root calculations is very good for long waves. For shorter waves the accuracy is still acceptable even without special treatment of the series. The root $\varkappa_{-1}$ is calculated with accuracy $10^{-15}$ and the roots on the imaginary axis with the accuracy $10^{-16}$.

The strain distribution around the cylinder in incident waves is important to estimate the possibility for the ice to be broken due to the wave-structure interaction. The yield strain for ice, $\epsilon_{r}^{*}$, is estimated as $8 \times 10^{-5}$, see [1]. On the contact line of the cylinder frozen in ice, only the radial strain component, $\epsilon_{r}(\theta, t)=0.5 h_{i} w_{r r}(b, \theta, t)$, is not equal to zero. Within the linear model of the present study, the strain reads

$$
\epsilon_{r}(\theta, t)=\frac{h_{i}}{2 H^{2}} \sum_{m=0}^{\infty} \Re\left[L_{m}(t)\left(\frac{L_{1}^{(m)}}{L_{0}^{(m)}}-\varkappa_{0}^{2}\right)\right] \cos (m \theta),
$$

where $L_{0}^{(m)}$ and $L_{1}^{(m)}$ are given by (91).

The amplitudes of the radial strains as functions of the polar angle $\theta$ are shown in Fig. 3 for the radius of the cylinder $b=2 \mathrm{~m}$, wave amplitude of $1 \mathrm{~cm}$, the non-dimensional wave number $k H=1$ in Fig. $3 a$ and $k H=0.7$ in Fig. $3 b$, and different thicknesses of the ice cover. The wave length, $\lambda=94.25 \mathrm{~m}$ for $k H=1$ and $\lambda=134.64 \mathrm{~m}$ for $k H=0.7$, is much greater than the cylinder radius, 

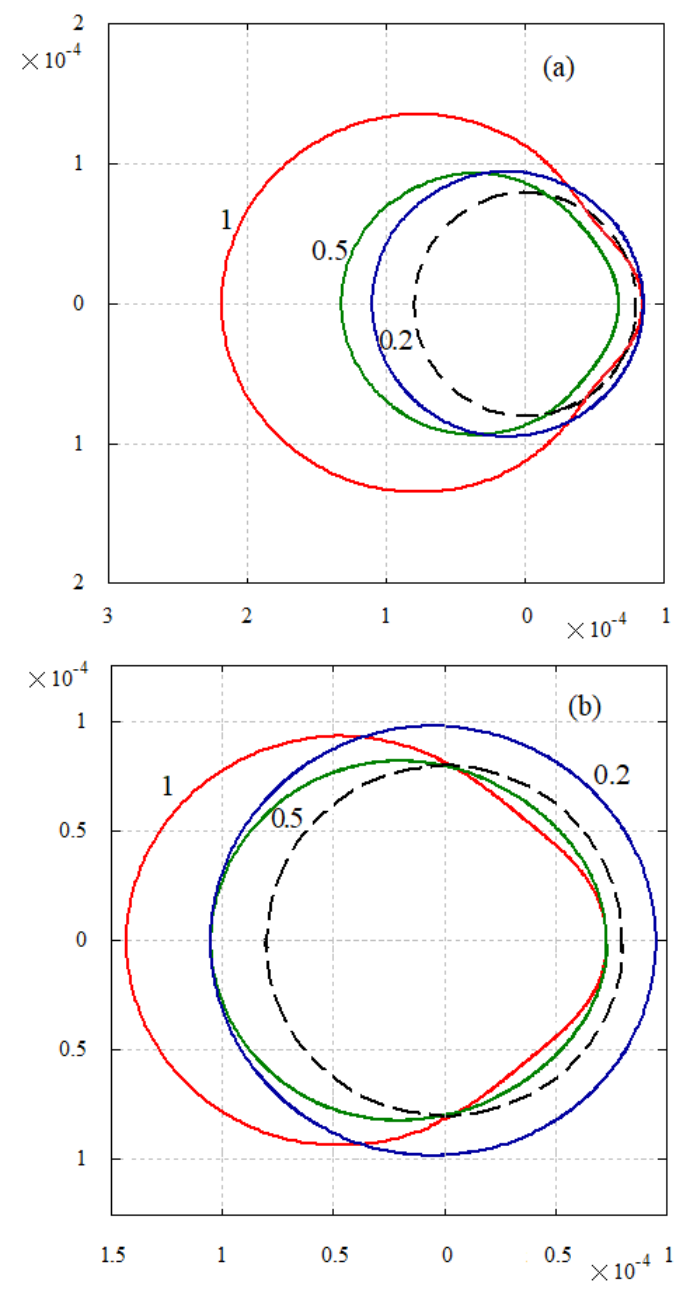

Fig. 3 The radial strain amplitudes at the contact line as functions of the angular coordinate $\theta$ for $H=15 \mathrm{~m}, b=2 \mathrm{~m}, A=1 \mathrm{~cm}$, the ice thickness $h_{i}=0.2,0.5,1 \mathrm{~m}$, and (a) $k H=1$; (b) $k H=0.7$.

The dashed circular line is for the yield strain $8 \times 10^{-5}$.

$b=2 \mathrm{~m}$, in these computations. It is seen that the strains are greater and more non-uniform for thicker ice. The stresses are greater than the yield stress $\epsilon_{r}^{*}$ in all cases. Note that the strains depend linearly on the wave amplitude $A$. Then the wave of $94 \mathrm{~m}$ length does not damage the ice contact with the cylinder for $h_{i}=1 \mathrm{~m}$ if the wave amplitude is smaller than $4 \mathrm{~mm}$, see Fig $3 a$. However, this rule works only if the strains in the incident wave are smaller than the yield strain. In the sinusoidal incident wave (8), the maximum strain is $2 \pi^{2} h_{i} A / \lambda^{2}$. For ice $1 \mathrm{~m}$ thick this maximum is smaller than the yield strain $\epsilon_{r}^{*}$ if $A(\mathrm{~m})<4 \times 10^{-6} \lambda^{2}(\mathrm{~m})$. For example, in the conditions of the present calculations, the amplitude of the incident wave cannot be larger than $35 \mathrm{~mm}$ for $\lambda=94 \mathrm{~m}$. In other words, a hydroelastic wave of length $94 \mathrm{~m}$ can propagate in ice cover with $h_{i}=1 \mathrm{~m}$ only if its amplitude is below $35 \mathrm{~mm}$. The waves of larger amplitude do not exist because they would break the ice cover. Interestingly, the linearisation parameter of the problem $k A$ cannot then be larger than $\epsilon_{r}^{*} \lambda /\left(\pi h_{i}\right)$ and, therefore, nonlinear effects might be important for very long waves and/or thin ice cover. For example, for $h_{i}=1 \mathrm{~m}$, we find that propagating waves exist with $k A<0.1$ if the wave length is larger than $250 \mathrm{~m}$. Therefore, for waves with length greater 
than $250 \mathrm{~m}$, their amplitudes can be large enough to justify nonlinear effects with the strains in the wave being below the yield strain.

In the problem of Section 5, the deflection, $w(b, \theta, t)$, of the free edge of the ice plate is given by equations $(13),(17),(18),(19),(86)$, and (87),

$$
w(b, \theta, t)=\sum_{m=0}^{\infty} \Re\left[L_{m}(t)\left(1-W_{m}(b)\right)\right] \cos (m \theta),
$$

where $W_{m}(b)$ is given by (86). The amplitude of the edge deflection is shown in Figure 4 in the polar coordinates for $H=15 \mathrm{~m}, h_{i}=1.5 \mathrm{~m}, b=10 \mathrm{~m}$, and incident wave amplitude $1 \mathrm{~cm}$. The incident wave propagates from left to right. The calculations were performed for three wavenumbers $k H=0.5,1$ and 1.2. It is seen that the deflection amplitude at the cylinder is close to the wave amplitude for long waves, which is shown by the unit dashed circle in the figure, and may exceed it twice for short waves. The shortest wave here is still more than five times longer than the diameter of the cylinder.

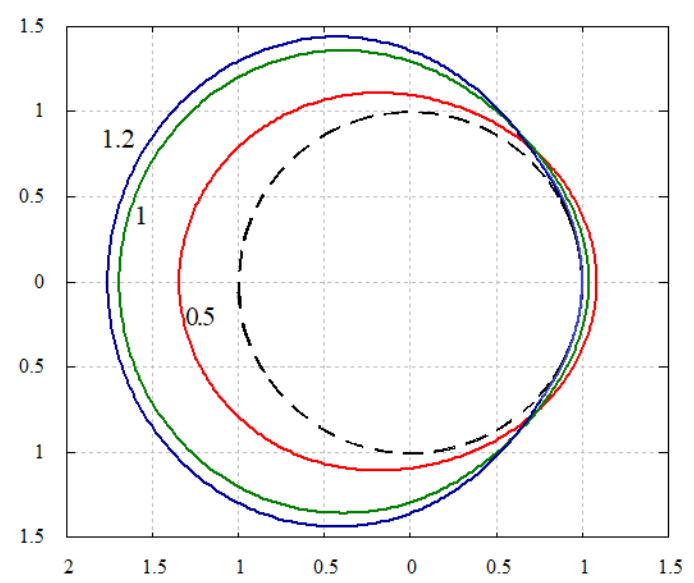

Fig. 4 Deflection (in $\mathrm{cm}$ ) of the free ice edge in polar coordinates for $k H=0.5,1.0,1.2$. The dashed circular line is for the deflection amplitude without the cylinder.

\section{Conclusion}

The problems of flexural-gravity waves and their interactions with a vertical circular cylinder have been investigated. The solutions of these problems were obtained by the method of vertical modes and by using the Weber integral transform. It was shown that the solutions by these two methods are identical for clamped conditions at the contact line between the ice sheet and the cylinder surface.

The method of the vertical modes is simpler than the integral transform method. However, the use of the orthogonality condition is not straightforward. One needs to prove that the solution obtained by the vertical-mode method does satisfy all equations and the boundary conditions either by direct substitution, see [3], or by comparing it with the solution obtained by another method. Justification of the vertical-mode solutions by direct substitution can be complicated and not feasible for vertical cylinders with non-circular cross sections [20] and for several cylinders frozen in ice. Even for a single circular cylinder with mixed boundary conditions at the contact line between the cylinder and the ice plate, where ice is clamped to the cylinder only along an interval, both the 
vertical-mode solution and its justification could be complicated. Also the vertical-mode method requires homogeneous conditions on the ice/water interface, which is an important limitation of the method.

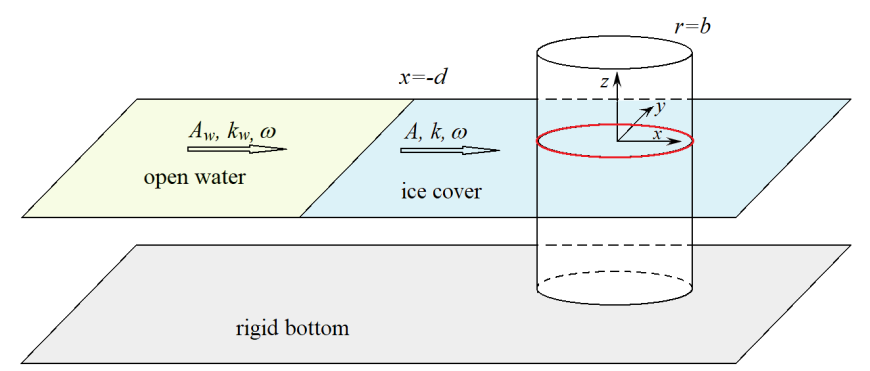

Fig. 5 A circular cylinder frozen in semi-infinite ice cover and exposed to an incident water wave.

The problem studied in this paper is simplified, see [25]. A more practical problem is sketched in Fig. 5, where a vertical cylinder is placed in an ice field at a distance from the edge of the ice half-plane. This problem can be solved approximately for a given amplitude $A_{w}$ and frequency $\omega$ of an incident water wave. By using the solution of the two-dimensional problem of a water wave diffracted by the edge of a floating elastic plate, we can find the amplitude $A$ and the wave number $k$ of the transmitted wave without the cylinder, see [26]. Note that the ice inertia is neglected in [26]. Then the transmitted flexural-gravity wave is considered as the incident wave for the cylinder, and the wave/cylinder interaction problem is solved by the methods of this paper. Let the distance $d$ of the cylinder from the edge of the ice field be much greater than the water depth $H$ and the radius of the cylinder $b$. The waves diffracted by the cylinder interact with the edge of the ice field and generate secondary incident waves. These secondary waves can be neglected if the cylinder is far enough from the ice edge.

\section{ACKNOWLEDGEMENTS}

This work was supported in parts by EPSRC Grant Number EP/K032208/1. SM acknowledges the support of the National Research Foundation of Korea (NRF) grant funded by the Korea Government (MEST) through GCRC-SOP. TK acknowledges the support from the grants RFBR 16-08-00291 "Hydroelastic and thermodynamic effects with interaction of poroelastic ice and structures". AK and TK acknowledge the support from Simons Foundation during their work at the Isaac Newton Institute for Mathematical Sciences in August-December 2017. The authors would like to thank the Isaac Newton Institute for Mathematical Sciences for support and hospitality during the programme "Mathematics of sea ice phenomena" when this study started.

\section{References}

1. Brocklehurst, P., Korobkin, A. A., and Parau, E. I. (2011). Hydroelastic wave diffraction by a vertical cylinder. Philosophical Transactions of the Royal Society of London A: Mathematical, Physical and Engineering Sciences, 369(1947), 2832-2851.

2. Bhattacharjee, J., and Soares, C. G. (2012). Flexural gravity wave over a floating ice sheet near a vertical wall. Journal of Engineering Mathematics, 75(1), 29-48.

3. Korobkin, A., Malenica S., and Khabakhpasheva, T. (2018) Interaction of flexural-gravity waves in ice cover with vertical walls. Philosophical Transactions of the Royal Society A: Mathematical, Physical and Engineering Sciences. (in press). 
4. Evans, D. V., and Porter, R. (2003). Wave scattering by narrow cracks in ice sheets floating on water of finite depth. Journal of Fluid Mechanics, 484, 143-165.

5. Lawrie, J.B. and Abrahams, I.D. (1999). An orthogonality relation for a class of problems with high-order boundary conditions; applications in sound-structure interaction. The Quarterly Journal of Mechanics and Applied Mathematics, 52(2), 161-181.

6. Lawrie, J.B. and Abrahams, I.D. (2002). On the propagation and scattering of fluid-structural waves in a three-dimensional duct bounded by thin elastic walls. In IUTAM Symposium on Diffraction and Scattering in Fluid Mechanics and Elasticity (pp. 279-288). Springer Netherlands.

7. Manam, S.R., Bhattacharjee, J. and Sahoo, T. (2006). Expansion formulae in wave structure interaction problems. Proceedings of the Royal Society of London A: Mathematical, Physical and Engineering Sciences (Vol. 462, No. 2065, pp. 263-287). The Royal Society.

8. Mandal, S., Sahoo, T. and Chakrabarti, A. (2015). A note on convergence of expansion formula for wave structure interaction problems. In 30th International Workshop on the Water Waves and Floating Bodies (IWWWFB).

9. Brocklehurst, P., Korobkin, A.A. and Parau, E.I. (2010). Interaction of hydro-elastic waves with a vertical wall. Journal of Engineering Mathematics, 68(3), pp.215-231.

10. Brocklehurst, P. (2012). Hydroelastic waves and their interaction with fixed structures. Doctoral dissertation, University of East Anglia.

11. Korobkin, A.A., Khabakhpasheva, T.I. and Papin, A.A. (2014). Waves propagating along a channel with ice cover. European Journal of Mechanics-B/Fluids, 47, pp.166-175.

12. Batyaev, E.A. and Khabakhpasheva, T.I. (2015). Hydroelastic waves in a channel covered with a free ice sheet. Fluid Dynamics, 50(6), pp.775-788.

13. Shishmarev, K., Khabakhpasheva, T. and Korobkin, A. (2016). The response of ice cover to a load moving along a frozen channel. Applied Ocean Research, 59, pp.313-326.

14. Malenica, S. and Korobkin, A. A. (2003) Water wave diffraction by vertical circular cylinder in partially frozen sea. In Proc. 18th International Workshop on Water Waves and Floating Bodies, Le Croisic, France.

15. Bennetts, L.G. (2007). Wave scattering by ice sheets of varying thickness. Doctoral dissertation, University of Reading.

16. Squire, V., Hosking, R. J., Kerr, A. D. and Langhorne, P. (2012). Moving loads on ice plates (Vol. 45). Springer Science \& Business Media.

17. Timokhov, L.A. and Heisin, D.E. (1987). Dynamics of Sea Ice. Gidrometeoizdat, Leningrad, 272 pp. (in Russian).

18. MacCamy, R. C. and Fuchs, R. A. (1954). Wave forces on piles: A diffraction theory. Report No. 69, U.S. Army Corps of Engineers, Beach Erosion Board, Washington, D.C.

19. Abramowitz, M. and Stegun, I. A. (1964). Handbook of mathematical functions: with formulas, graphs, and mathematical tables (Vol. 55). Courier Corporation.

20. Disibuyuk N. B., Korobkin A. A. and Yilmaz, O., (2017). Linear wave interaction with a vertical cylinder of arbitrary cross section: an asymptotic approach. Journal of Waterway, Port, Coastal, and Ocean Engineering, 143(5), 04017028.

21. Friedman, B. (1956). Principles and techniques of applied mathematics. Courier Dover Publications.

22. Emmerhoff, O. J. and Sclavounos, P. D. (1992). The slow-drift motion of arrays of vertical cylinders. Journal of Fluid Mechanics, 242, 31-50.

23. Newman, J. N. (1996). The second-order wave force on a vertical cylinder. Journal of Fluid Mechanics, 320, 417-443.

24. Leissa, A. W. (1969). Vibration of plates, Ohio State University Columbus. 
25. Korobkin, A. A., Părău, E. I. and Vanden-Broeck, J-M. (2011). The mathematical challenges and modelling of hydroelasticity. Philosophical Transactions of the Royal Society A: Mathematical, Physical and Engineering Sciences, 369(1947), 2803-2812.

26. Tkacheva, L. A. (2001). Surface wave diffraction on a floating elastic plate. Fluid Dynamics, 36(5), 776-789. 